\title{
Heavy quark free energies for three quark systems at finite temperature
}

\author{
Kay Hübner and Frithjof Karsch \\ Physics Department, Brookhaven National Laboratory, Upton, NY 11973, USA \\ Olaf Kaczmarek and Oliver Vogt \\ Fakultät für Physik, Universität Bielefeld, D-33615 Bielefeld, Germany
}

(Dated: October 22, 2018)

\begin{abstract}
We study the free energy of static three quark systems in singlet, octet, decuplet and average color channels in the quenched approximation and in 2-flavor QCD at finite temperature. We show that in the high temperature phase singlet and decuplet free energies of three quark systems are well described by the sum of the free energies of three diquark systems plus self energy contributions of the three quarks. In the confining low temperature phase we find evidence for a Y-shaped flux tube in $\mathrm{SU}(3)$ pure gauge theory, which is less evident in 2-flavor QCD due to the onset of string breaking. We also compare the short distance behavior of octet and decuplet free energies to the free energies of single static quarks in the corresponding color representations.
\end{abstract}

PACS numbers:

\section{INTRODUCTION}

The study of baryonic systems composed of three static quarks sheds light on the internal structure of the baryon. Lattice calculations have been carried out addressing this question for quite some time now $1,2,2,3,4,5,6,6,7,8]$. Of particular interest is the question whether a genuine three body force exists between the quarks below the critical temperature and how the system behaves at finite temperature. At zero temperature the Y-string shape of the color flux tube in baryonic systems is supported by recent calculations in lattice QCD [4, 6, 8]. At finite temperature work so far has concentrated on simulations using maximal abelian gauge [6, 7], showing the existence of a Y-shaped string as well. The Y-Ansatz is also supported by results obtainde with the field correlator method [9].

Recently, there have been speculations that hadronic bound states, e. g. also colored $Q Q Q$-states, may still exist in the high temperature deconfined phase of QCD and that they could be responsible for the observed strong interactions in the QGP near $T_{c}[10]$. This makes it interesting to analyze the force in three quark systems also in non-singlet color configurations that might exist in an overall color neutral thermal medium.

In this paper, we examine the question of the flux tube shape of the $Q Q Q$-singlet state below the critical temperature in the quenched approximation of QCD and discuss the appearance of string breaking in 2-flavor QCD. Moreover, above $T_{c}$ we explore the relation between the free energies in different color channels of the baryonic system and the free energies of $Q Q$-subsystems, and analyze the screening of octet and decuplet free energies at small distances.

This paper is organised as follows: In section II we present the basic observables we use for calculations in different color channels of the heavy three quark system in terms of correlation functions of thermal Wilson lines. In section III we discuss the perturbative behavior of free energies of three quark systems at short distances. In sec- tion [V] we present the details of our simulation, including calculation techniques, gauge fixing and renormalisation procedures. In section $\mathrm{V}$ we discuss the behavior of $Q Q Q$-free energies obtained in $\mathrm{SU}(3)$ pure gauge theory on $32^{3} \times 4,8$ lattices and compare results above $T_{c}$ with free energies of $Q Q$-systems. Furthermore, we examine the string shape below $T_{c}$. Section VI is devoted to a discussion of our results in 2-flavor QCD. In section VII we analyze the screening of octet and decuplet free energies at small distances. Finally, in section VIII we conclude.

\section{STATIC THREE QUARK SYSTEMS IN DIFFERENT COLOR REPRESENTATIONS}

In the following we will construct observables for the free energies of static three quark systems in different color channels and introduce the corresponding relation to the expectation values of correlation functions of thermal Wilson lines. Since we are interested in QCD, we restrict ourselves to examine the $\mathrm{SU}(3)$ gauge group.

The state of a three quark system as the product of irreducable representations of three quarks in color space can be decomposed into symmetry states

$$
3 \otimes 3 \otimes 3=1 \oplus 8 \oplus 8^{\prime} \oplus 10 .
$$

The singlet is totally anti-symmetric, the first octet antisymmetric in the first and second, the second octet in the second and third component and the decuplet is totally symmetric.

We now construct observables for static three quark systems represented by suitable combinations of Wilson lines. The derivation is similiar to that for two quark systems [11, 12], but more elaborate. We start by defining the thermal Wilson line

$$
L(\mathbf{x})=\prod_{x_{4}=0}^{N_{\tau}-1} U_{4}\left(\mathbf{x}, x_{4}\right) .
$$


The Polyakov loop is then obtained by $l(\mathbf{x})=\operatorname{Tr} L(\mathbf{x})$, where the trace is normalised such that $\operatorname{Tr} \mathbf{1}=3$. For the three quark correlation function of thermal Wilson lines ${ }^{1} L_{1}, L_{2}, L_{3}$, we have

$$
L_{1} L_{2} L_{3}=\sum_{s} C_{Q Q Q}^{s}\left(\mathbf{x}_{1}, \mathbf{x}_{2}, \mathbf{x}_{3}\right) P_{s}
$$

where $s \in\left\{1,8,8^{\prime}, 10\right\}$ stands for the symmetry states, $P_{s}$ for the projectors on these states and $C_{Q Q Q}^{s}$ for the three point correlator of the thermal Wilson line belonging to that symmetry state. Here we have suppressed the dependence on the temperature $T$.

Denoting the components of the thermal Wilson lines $L_{1}^{i l}, L_{2}^{j m}, L_{3}^{k n}$, where $i, j, k, l, m, n=1,2,3$, we find the projectors $P_{s}$ by using the Fierz-identity

$$
\begin{aligned}
P_{1} & =\frac{1}{6} \sum_{\sigma(l, m, n)} \epsilon^{l m n} \delta^{i l} \delta^{j m} \delta^{k n} \\
P_{8} & =\frac{1}{3} \sum_{\sigma(l, m, n)} \epsilon^{l m} \delta^{i l} \delta^{j m} \delta^{k n} \\
P_{8^{\prime}} & =\frac{1}{3} \sum_{\sigma(l, m, n)} \epsilon^{n m} \delta^{i l} \delta^{j m} \delta^{k n} \\
P_{10} & =\frac{1}{6} \sum_{\sigma(l, m, n)} \delta^{i l} \delta^{j m} \delta^{k n},
\end{aligned}
$$

where the sums are over all permutations $\sigma$ of the indices. The $P_{s}$ satisfy the usual relations for projectors

$$
P_{s}^{2}=P_{s}, \quad \sum_{s} P_{s}=1 \quad \text { und } \quad P_{s} P_{t}=0
$$

for $s \neq t$ and $s, t \in\left\{1,8,8^{\prime}, 10\right\}$. The desired three quark correlators in the different color channels are now obtained by applying

$$
C_{Q Q Q}^{s}=\frac{\operatorname{Tr}\left(P_{s} L_{1} L_{2} L_{3}\right)}{\operatorname{Tr} P_{s}} .
$$

\footnotetext{
${ }^{1}$ For convenience we write $L_{i}$ instead of $L\left(\mathbf{x}_{i}\right)$.
}

We find

$$
\begin{aligned}
& C_{Q Q Q}^{1} \\
& =\frac{1}{6}\left(27 \operatorname{Tr} L_{1} \operatorname{Tr} L_{2} \operatorname{Tr} L_{3}-9 \operatorname{Tr} L_{1} \operatorname{Tr}\left(L_{2} L_{3}\right)\right. \\
& -9 \operatorname{Tr} L_{2} \operatorname{Tr}\left(L_{1} L_{3}\right)-9 \operatorname{Tr} L_{3} \operatorname{Tr}\left(L_{1} L_{2}\right) \\
& \left.+3 \operatorname{Tr}\left(L_{1} L_{2} L_{3}\right)+3 \operatorname{Tr}\left(L_{1} L_{3} L_{2}\right)\right) \\
& C_{Q Q Q}^{8} \\
& =\frac{1}{24}\left(27 \operatorname{Tr} L_{1} \operatorname{Tr} L_{2} \operatorname{Tr} L_{3}+9 \operatorname{Tr} L_{1} \operatorname{Tr}\left(L_{2} L_{3}\right)\right. \\
& \left.-9 \operatorname{Tr} L_{3} \operatorname{Tr}\left(L_{1} L_{2}\right)-3 \operatorname{Tr}\left(L_{1} L_{3} L_{2}\right)\right) \\
& C_{Q Q Q}^{8^{\prime}} \\
& =\frac{1}{24}\left(27 \operatorname{Tr} L_{1} \operatorname{Tr} L_{2} \operatorname{Tr} L_{3}+9 \operatorname{Tr} L_{3} \operatorname{Tr}\left(L_{1} L_{2}\right)\right. \\
& \left.-9 \operatorname{Tr} L_{1} \operatorname{Tr}\left(L_{2} L_{3}\right)-3 \operatorname{Tr}\left(L_{1} L_{2} L_{3}\right)\right) \\
& C_{Q Q Q}^{10} \\
& =\frac{1}{60}\left(27 \operatorname{Tr} L_{1} \operatorname{Tr} L_{2} \operatorname{Tr} L_{3}+9 \operatorname{Tr} L_{1} \operatorname{Tr}\left(L_{2} L_{3}\right)\right. \\
& +9 \operatorname{Tr} L_{2} \operatorname{Tr}\left(L_{1} L_{3}\right)+9 \operatorname{Tr} L_{3} \operatorname{Tr}\left(L_{1} L_{2}\right) \\
& \left.+3 \operatorname{Tr}\left(L_{1} L_{2} L_{3}\right)+3 \operatorname{Tr}\left(L_{1} L_{3} L_{2}\right)\right) \text {. }
\end{aligned}
$$

Finally we obtain for the color averaged correlator of the three quark system, $C_{Q Q Q}^{\mathrm{av}}$, the relation

$$
\begin{aligned}
C_{Q Q Q}^{\mathrm{av}} & =\frac{1}{27} C_{Q Q Q}^{1}+\frac{8}{27} C_{Q Q Q}^{8}+\frac{8}{27} C_{Q Q Q}^{8^{\prime}}+\frac{10}{27} C_{Q Q Q}^{10} \\
& =\frac{1}{27} \operatorname{Tr} L_{1} \operatorname{Tr} L_{2} \operatorname{Tr} L_{3} .
\end{aligned}
$$

We note that the two octet correlators $C_{Q Q Q}^{8}$ and $C_{Q Q Q}^{8^{\prime}}$ are the same when calculated on the lattice. The free energy of the symmetry state $s$ can be obtained from the correlator in the usual way

$$
F_{Q Q Q}^{s}(T)=-T \ln \left\langle C_{Q Q Q}^{s}(T)\right\rangle,
$$

where $\langle\cdot\rangle$ stands for thermal averages taken after gauge fixing. We have suppressed the position dependence on both sides of (15).

In the following section we will discuss briefly the behavior of the free energies of the three quark system for small couplings and short distances.

\section{F $_{Q Q Q}^{s}$ AT SHORT DISTANCES}

In the perturbative expansion of the free energy of $Q Q Q$-systems the contribution of the three gluon vertex vanishes for symmetry reasons [13]. Therefore, neglecting self energy contributions, to order $g^{4}$ the free energy 
$F_{Q Q Q}^{s}$ decomposes into the sum of three diquark free energies $F_{Q Q}^{t}$, which can be in an anti-symmetric antitriplet $(t=\overline{3})$ or in a symmetric sextet $(t=6)$ state. The behavior of these diquark free energies at small distances to lowest order are Coulombic

$$
F^{t}(R, T)=C_{2}(t) \frac{\alpha}{R},
$$

where $\alpha=\frac{g^{2}}{4 \pi}$ and $R$ is the separation of the static quarks in the $Q Q$-system. The Casimir factor $C_{2}=\operatorname{Tr}\left(t_{1}^{a} t_{2}^{a}\right)$ depends on the color channel $t$ the diquark system is in and can be found in table प. In order to obey the permutation relations given for the $Q Q Q$-system in (4) - (7), the $Q Q Q$-singlet state must be composed of $Q Q$-anti-triplets, the $Q Q Q$-decuplet of $Q Q$-sextets and the $Q Q Q$-octets are a mixture of $Q Q$-anti-triplets and -sextets. Thus, we have for small distances

$$
\begin{aligned}
& F_{Q Q Q}^{1}(\mathbf{R}, T)=\sum_{i<j}-\frac{2}{3} \frac{\alpha}{R_{i j}}+\tilde{k}_{1}(T) \\
& F_{Q Q Q}^{8}(\mathbf{R}, T)=-\frac{2}{3} \frac{\alpha}{R_{12}}-\frac{1}{6} \frac{\alpha}{R_{13}}+\frac{1}{3} \frac{\alpha}{R_{23}}+\tilde{k}_{8}(T) \\
& F_{Q Q Q}^{8^{\prime}}(\mathbf{R}, T)=\frac{1}{3} \frac{\alpha}{R_{12}}+\frac{1}{6} \frac{\alpha}{R_{13}}-\frac{2}{3} \frac{\alpha}{R_{23}}-+\tilde{k}_{8^{\prime}}(T) \\
& F_{Q Q Q}^{10}(\mathbf{R}, T)=\sum_{i<j} \frac{1}{3} \frac{\alpha}{R_{i j}}+\tilde{k}_{10}(T),
\end{aligned}
$$

where $R_{i j}$ denotes the distance between the $i$ th and $j$ th quark (see sec. IVB for details), $\mathbf{R}=\left(R_{12}, R_{13}, R_{23}\right)$. The self energy contributions $\tilde{k}_{i}$ with $i=1,8,8^{\prime}, 10$ are temperature dependent and at small separations related to the free energy of a single static quark in the corresponding color state, $F_{Q}^{(s)}(T)$ (see sec. VII for details). Thus $\tilde{k}_{1}(T)=0, \tilde{k}_{8}(T)=\tilde{k}_{8^{\prime}}(T)=F_{Q}^{(8)}(T)$ and $\tilde{k}_{10}(T)=F_{Q}^{(10)}(T)$. As can be seen easily from (17) and (20), the singlet state of the $Q Q Q$-system is attractive, because the $Q Q$-antitriplet is, and the decuplet state is repulsive, as the $Q Q$-sextet is. Moreover, the $Q Q Q$-singlet free energy is temperature independent at small distances like the $Q \bar{Q}$-singlet free energy is $[15]$. For the octet channels the situation is more complicated due to the contribution of both anti-triplet and sextet free energies. We can, however, compute the lowest order behavior of the $Q Q Q$ free energies for equilateral geometries for all color channels. Table \ summarizes the Casimir factors for the free energies of the different color channels for all the quark systems $Q \bar{Q}, Q Q$ and $Q Q Q$. One recovers the lowest order behavior of the free energy

\begin{tabular}{|c|c|c|c|c|c|}
\hline stem & 1 & $\overline{3}$ & 6 & 8 & 10 \\
\hline$\overline{Q \bar{Q}}$ & $-4 / 3$ & & & $+1 / 6$ & \\
\hline$Q Q$ & \multicolumn{4}{|c|}{$-2 / 3+1 / 3$} & \\
\hline$Q Q Q^{\dagger}$ & -2 & & & $-1 / 2^{\prime}$ & +1 \\
\hline
\end{tabular}
in some symmetry state $t$ (equilateral geomtries for the $Q Q Q$-systems only) that depends on the separation of the static quarks by using (16), where $R$ is now the separation of the static quarks in the $Q \bar{Q}$ - and $Q Q$-systems
TABLE I: Casimir factor for color symmetry state $t, C_{2}(t)=$ $\operatorname{Tr}\left(T_{1}^{a} T_{2}^{a}\right)$.

$\dagger$ equilateral geometries, ${ }^{\star}$ average of both octets.

and the edge length of the equilateral geometry in the $Q Q Q$ case. See sec. IVB for details on the geometric configuration of the three static quarks. We see, that the average of both $Q Q Q$-octet free energies (which is accessible to lattice calculations) for equilateral geometries is expected to be weakly attractive.

At larger distances, the pairwise interactions of the static quarks should receive temperature dependent contributions and the Coulomb terms in (17) - (20) have to be replaced by the full diquark free energies $F_{Q Q}^{t}(R, T)$ of the corresponding color channel $t$. In this case, (17) (20) generalize to

$$
\begin{aligned}
F_{Q Q Q}^{1}(\mathbf{R}, T)= & \sum_{i<j} F_{Q Q}^{\overline{3}}\left(R_{i j}, T\right)+k_{1}(T) \\
F_{Q Q Q}^{8}(\mathbf{R}, T)= & F_{Q Q}^{\overline{3}}\left(R_{12}\right)+\frac{1}{4} F_{Q Q}^{\overline{3}}\left(R_{13}\right) \\
& +F_{Q Q}^{6}\left(R_{23}\right)+k_{8}(T) \\
F_{Q Q Q}^{8^{\prime}}(\mathbf{R}, T)= & F_{Q Q}^{6}\left(R_{12}\right)-\frac{1}{4} F_{Q Q}^{\overline{3}}\left(R_{13}\right) \\
& +F_{Q Q}^{\overline{3}}\left(R_{23}\right)+k_{8^{\prime}}(T) \\
F_{Q Q Q}^{10}(\mathbf{R}, T)= & \sum_{i<j} F_{Q Q}^{6}\left(R_{i j}, T\right)+k_{10}(T),
\end{aligned}
$$

where the $k_{i}(T)$ with $i=1,8,8^{\prime}, 10$ account for the self energy contributions not included in the diquark free energies. It was shown in [14] that the residual $T$ dependence of the $Q Q$-anti-triplet free energy at small distances in the deconfined phase can be removed by substracting the free energy of a single static quark $F_{Q}(T) \equiv \frac{1}{2} \lim _{R \rightarrow \infty} F_{Q \bar{Q}}^{1}(R, T)$. We therefore expect $k_{1}(T)=-3 F_{Q}(T)$, which guarantees the cancelation of self energy contributions stemming from $F_{Q Q}^{\overline{3}}$, leaving $F_{Q Q Q}^{1}$ temperature independent at small separations. Moreover, at large separations, the self energy contributions for the $Q Q Q$-system should be independent from the particular color channel the system is in, leading to $k_{1}(T)=k_{8}(T)=k_{8^{\prime}}(T)=k_{10}(T)=-3 F_{Q}(T)$. We will show in sections VIVI and VII that this reasoning is indeed correct for temperatures $T>T_{c}$. Indeed, we will show that the decomposition of interactions in 3-quark systems in terms of 2-quark interactions as suggested by 


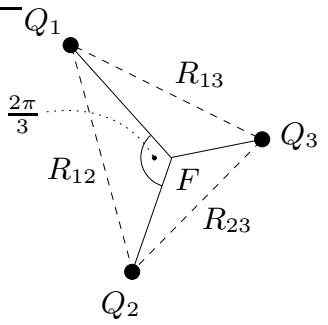

FIG. 1: Inter quark distances. $F$ is the Fermat point of the triangle.

(21)-(24) holds for $T>T_{c}$.

We finally note, that the $Q Q Q$-octet free energy obtained from the lattice is the average of $F_{Q Q Q}^{8}$ and $F_{Q Q Q}^{8^{\prime}}$, as will be shown in sec. IV Consequently, we expect the lattice $Q Q Q$-octet free energy for equilateral geometries to obey

$$
\begin{aligned}
F_{Q Q Q}^{8}(R, T)= & \frac{3}{2}\left(F_{Q Q}^{\overline{3}}(R, T)+F_{Q Q}^{6}(R, T)\right) \\
& -3 F_{Q}(T),
\end{aligned}
$$

where $R$ is the edge length of the equilateral triangle.

\section{SIMULATION DETAILS}

We will now present the details of our simulation.

\section{A. Simulation}

We used gauge field configurations generated on $32^{3} \times 4$ and $32^{3} \times 8$ lattices in pure gauge theory with the tree level-Symanzik improved gauge action at several couplings above and below the critical coupling [16, 17]. In 2-flavor staggered QCD we reexamined configurations on a $16^{3} \times 4$ lattice for several different couplings at bare quark mass $m / T=0.4$, where for fermions the p4-action and for the gauge fields again the tree level-Symanzik improved gauge action were used [18, 19, 20]. The scale was set using the string tension, $\sigma$, at $T=0$ following [21] and use it to express all dimensionful observables. On all configurations we calculated the three point correlation functions of Polyakov loops (10) - (13), (14) in a manner explained below.

The operators in (10) - (13) are not manifestly gauge independent and therefore a gauge fixing procedure must be applied to our gauge configurations. We use Coulomb gauge for our calculations, in which the singlet free energies of the $Q \bar{Q}$-system are related to a gauge independent definition in terms of dressed Polyakov lines 22]. Note that an operator dependence might still persist within this definition [23].

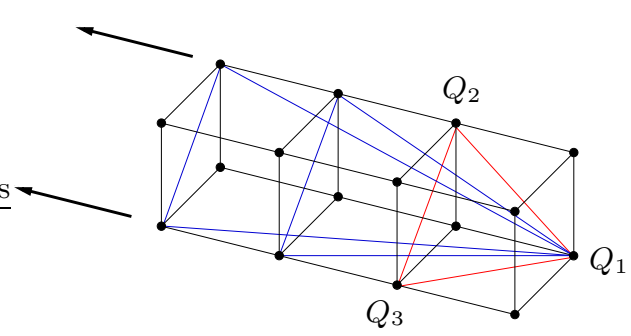

FIG. 2: Calculation of the three point correlation function of the Polyakov loop.

\section{B. Calculation technique}

We first fix the notation for the geometries of the three quark system. In fig. 1 we show three quarks $Q_{i}$ forming a triangle and their distances $R_{i j}$, where $i, j=1,2,3$. The perimeter of the triangle is then simply given by

$$
P=\sum_{i<j} R_{i j}
$$

This is also the total length of a $\Delta$-shaped string, i. e. a string connecting the three quarks along the edges of the triangle. Another possible string shape is a Y-shaped string, where the flux tube emanetes from each of the three quarks and has a junction at the Fermat point $F$ of the triangle. The total length of such a Y-shaped string is

$$
L=\left[\frac{1}{2} \sum_{i<j} R_{i j}^{2}+2 \sqrt{3} A_{\Delta}\right]^{\frac{1}{2}},
$$

where $A_{\Delta}$ is the area of the triangle [6]. The inner angles at the vertices of the triangle are assumed to be smaller than $\frac{2 \pi}{3}$, which is the case for all triangles considered in this work. The angle between any two arms of the Yshaped flux tube is always $\frac{2 \pi}{3}$. For equilateral triangles we have the simple relation $P=\sqrt{3} L$. In this work we examine only isoscele triangles, where we set $R_{12}=$ $R_{13}=R_{s}$ to be the equally long edges and $R_{23}=R_{b}$ to be the length of the base edge. For equilateral triangles we have $R_{i j}=R$ for all $i, j=1,2,3$.

The three point correlation functions of the Polyakov loop are now obtained as follows (see fig. 21). At the positions of the $Q_{i}$, we calculate the correlation functions (10) - (13), (14) and compute the average of the correlation function for those $Q_{i}$ with the same $\left\{R_{i j}\right\}$. We obtain a new set of $\left\{R_{i j}\right\}$ by holding $Q_{1}$ fixed, whereas the two other vertices of the triangle $Q_{2}$ and $Q_{3}$ are moved simultanously one point of the lattice in one direction (here: to the left). The base edge $R_{b}$ connecting these to points preserves thereby its length, which is $R_{b} / a=n \sqrt{2}$, where $a$ denotes the lattice spacing and $1 \leq n<\frac{N_{\sigma}}{2}$ is an integer and describes the number of elementary cells the procedure starts with. The two other edges have equal lengths $R_{s} / a=\sqrt{m^{2}+n^{2}}$, where $m$ is another integer 


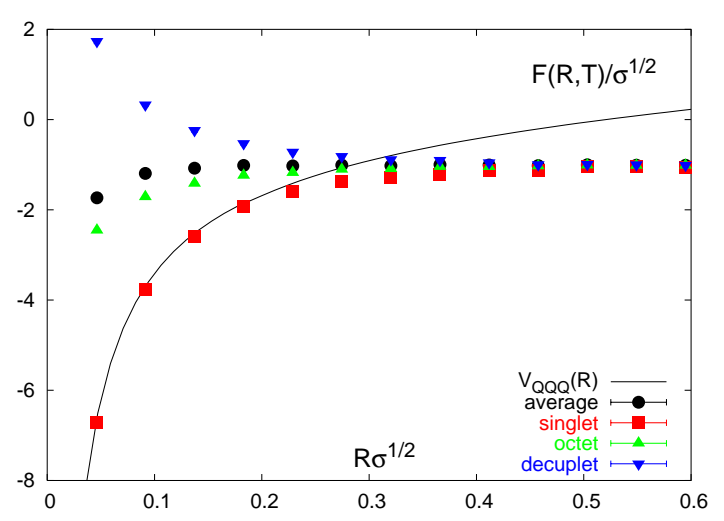

FIG. 3: Free energies in different color channels obtained on a $32^{3} \times 8$ lattice at $T / T_{c}=6$ from simulations in quenched QCD.

which runs between $n \leq m<\frac{N_{\sigma}}{2}$ for every $n$. Therefore for every $n$ we obtain one equilateral $(n=m)$ and several isosceles $\left(n<m<\frac{N_{\sigma}}{2}\right)$ triangles. We start with $n=1$ and repeat the procedure until $n=\frac{N_{\sigma}}{2}-1$. We apply this method in both directions of all three spatial dimensions before sweeping $Q_{1}$ over the entire spatial lattice. We will denote the configuration averages of the correlation functions (10) - (13) and (14) by $C_{Q Q Q}^{s}$ with $s=1,8,10$, av from now on.

\section{Renormalization}

We construct all correlation functions using renormalised thermal Wilson lines $L^{\text {ren }}(T)$, which are obtained from the bare ones calculated on lattices with temporal extend $N_{\tau}$ through multiplicative renormalisation,

$$
L^{\mathrm{ren}}(T)=\left(Z\left(g^{2}\right)\right)^{N_{\tau}} L\left(g^{2}, N_{\tau}\right),
$$

where $Z\left(g^{2}\right)$ is the multiplicative renormalisation constant determined in 15], $g$ is the bare coupling and $N_{\tau}$ is the temporal extent of the lattice $L$ is calculated on. The renormalization constants only depend on the bare coupling [24, 25] (and in addition on the bare quark masses in full QCD) and furthermore are equivalent at zero and finite temperature [26]. The renormalised three point correlation function of the thermal Wilson lines, $C^{\text {ren }}(T)$, is then obtained from the bare one, $C_{Q Q Q}\left(g^{2}, N_{\tau}\right)$, by

$$
C_{Q Q Q}^{\mathrm{ren}}(T)=\left(Z\left(g^{2}\right)\right)^{3 N_{\tau}} C_{Q Q Q}\left(g^{2}, N_{\tau}\right) .
$$

\section{RESULTS IN PURE GAUGE}

In this section we will analyze the behavior of three quark free energies in different color channels above $T_{c}$. We compare results with the perturbative expressions obtained in section 【II as well as with results obtained for

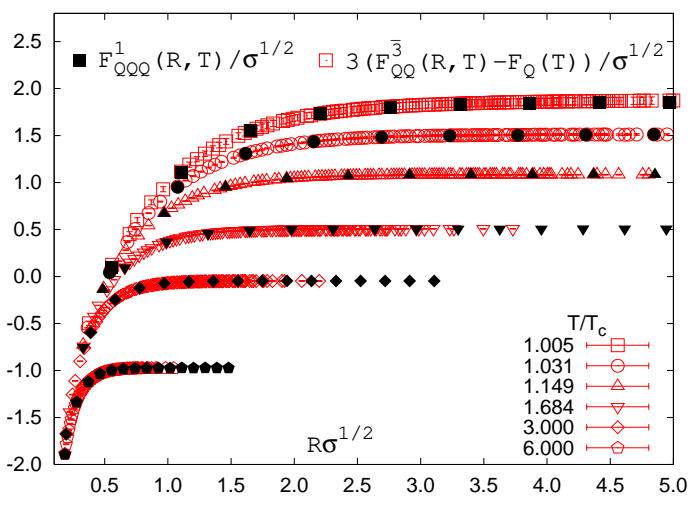

FIG. 4: $F_{Q Q Q}^{1}(R, T)$ and $3\left(F_{Q Q}^{3}(R, T)-F_{Q}(T)\right)$ above $T_{c}$ versus $R$, the edge length $R$ of the equilateral triangles and $Q Q$-distance, respectively.

$Q Q$-systems in different color channels. Data for the $Q Q$ free energies have been taken from [14]. Below $T_{c}$ we examine the string shape of the baryonic system and its structure in the different color channels.

\section{A. Color Channels}

In fig. 3 we show the free energies of three quark systems in different color channels and the average free energy for the $Q Q Q$-system for equilateral triangles of edge length $R$ calculated on a $32^{3} \times 8$ lattice at $T / T_{c}=6$. One can see clearly, that the singlet is strongly, the octet weaker attractive and the decuplet repulsive in agreement with the perturbative findings presented in sec. III. For large $R$ at a given temperature, all free energies in the different color channels approach a common value, i. e. the three quarks are screened independently of their color orientation. The singlet free energy becomes temperature independent at small distances and coincides with the baryonic $T=0$ potential, $V_{Q Q Q}(R)$, (see also fig. (4), which is related to the quark-antiquark potential at vanishing temperature by the ratio of the different Casimir operators, i. e. $V_{Q Q Q}(R)=\frac{3}{2} V_{Q \bar{Q}}(R)$ for $R \Lambda_{Q C D} \ll 1$. We obtain similiar results for all other temperatures above $T_{c}$. We will discuss the screening of octet and decuplet free energies at small distances in sec. VII]

\section{B. Free energies of equilateral geometries above $T_{c}$}

We now compare the free energies of the $Q Q Q$-system with the free energy of the $Q Q$-system above $T_{c}$. In fig.4 we show $F_{Q Q Q}^{1}(R, T)$ and $3 F_{Q Q}^{\overline{3}}(R, T)-3 F_{Q}(T)$ versus the edge length $R$ of the equilateral triangles and the $Q Q$ distance, respectively. According to (21) the second term is expected to be equal to the $Q Q Q$-singlet free energy at least at small distances, where genuine three body forces 


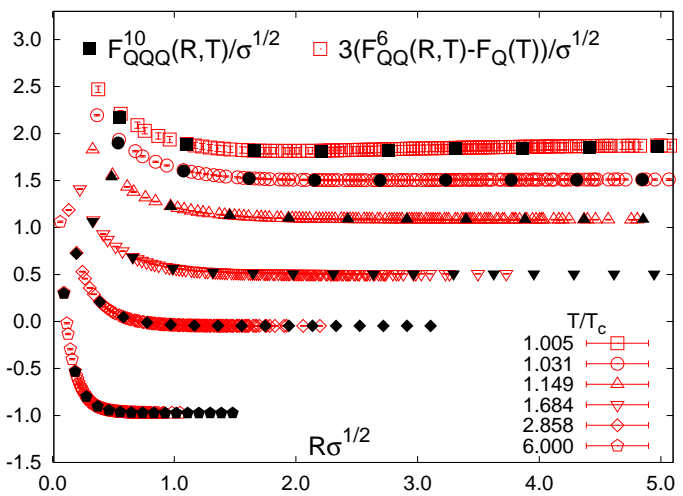

FIG. 5: $F_{Q Q Q}^{10}(R, T)$ and $3\left(F_{Q Q}^{6}(R, T)-F_{Q}(T)\right)$ above $T_{c}$ versus $R$, the edge length $R$ of the equilateral triangles and $Q Q$-distance, respectively.

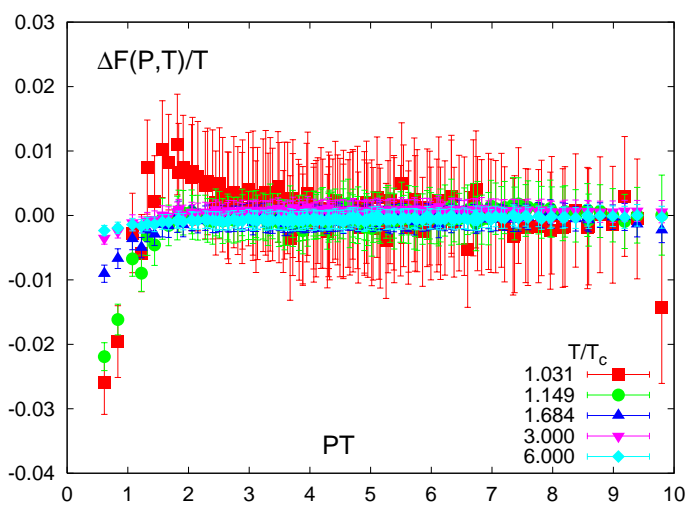

FIG. 6: $\Delta F(P, T)=F_{Q Q Q}^{1}(P, T)-\sum_{i} F_{Q Q}^{\overline{3}}\left(R_{i j}, T\right)+3 F_{Q}(T)$ above $T_{c}$ versus the perimeter $P$ for all geometries calculated.

are negligible. For all temperatures above $T_{c}$ we indeed see that $F_{Q Q Q}^{1}(R, T)$ and $3 F_{Q Q}^{\overline{3}}(R, T)-3 F_{Q}(T)$ coincide throughout the entire distance interval. In fig. 5 we show analogously $F_{Q Q Q}^{10}(R, T)$ and $3 F_{Q Q}^{6}(R, T)-3 F_{Q}(T)$. Again we observe that both observables do coincide. We obtain similar plots for $F_{Q Q Q}^{8}(R, T)$ in accordance with (25).

\section{Free energies of isosceles geometries above $T_{c}$}

In order to test whether the simple relation between free energies of a 3 quark system and the free energies of a 2 quark system plus self energy terms also holds for other geometries and temperatures above $T_{c}$, we calculate the difference $\Delta F(P, T)=F_{Q Q Q}^{1}(P, T)-\sum_{i<j} F_{Q Q}^{\overline{3}}\left(R_{i j}, T\right)+$ $3 F_{Q}$. This is shown in fig. 6. If (21) also holds for these geometries, then $\Delta F(P, T)$ should vanish, which is fullfilled to a very good degree for all perimeters except the smallest ones. Again the same holds true also for the $Q Q Q$-decuplet free energy as is evident from fig. 7. This is possibly due to the effect, that the self energy of the

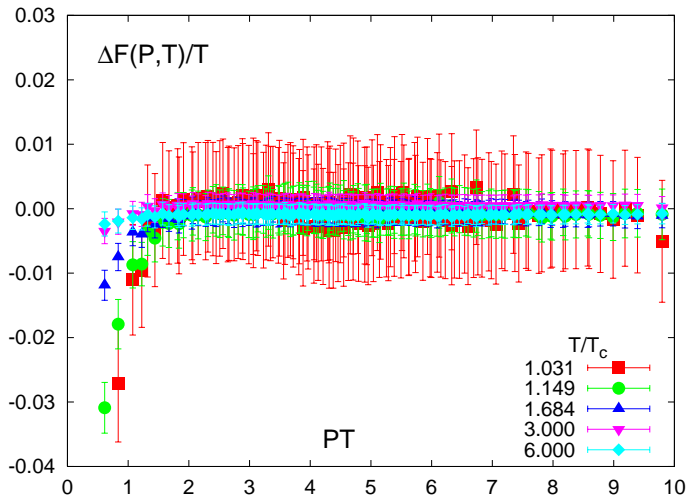

FIG. 7: $\Delta F(P, T)=F_{Q Q Q}^{10}(P, T)-\sum_{i<j} F_{Q Q}^{6}\left(R_{i j}, T\right)+$ $3 F_{Q}(T)$ above $T_{c}$ versus the perimeter $P$ for all geometries calculated.

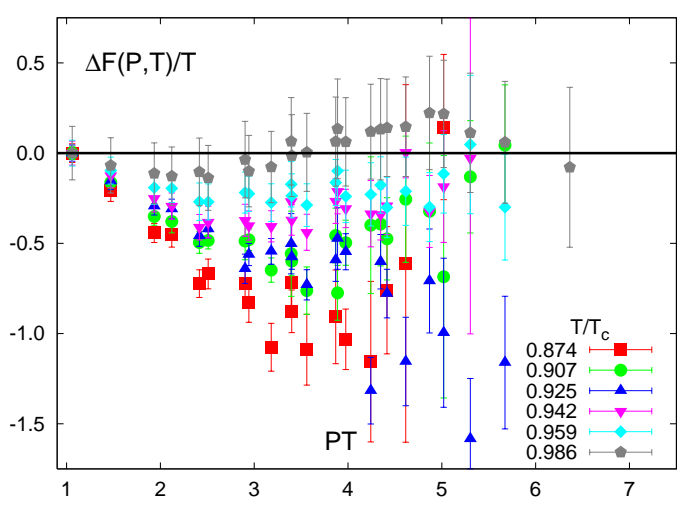

FIG. 8: $\Delta F(P, T)=F_{Q Q Q}^{1}(P, T)-\sum_{i<j} F_{Q Q}^{\overline{3}}\left(R_{i j}, T\right)$ below $T_{c}$ versus the perimeter $P$ for all geometries calculated. $\Delta F\left(P_{\min }, T\right)$ has been set to zero, where $P_{\min }$ is the smallest perimeter calculated.

system is no longer the sum of the self energy of the individual static quarks at short distances but rather that of a single static quark in the corresponding color representation (see sec. VII).

Having now established that (21) and (24) hold for all isoscele geometries above $T_{c}$ calculated in this work, it is clear that the screening length of the three quark system in the singlet state is the same as that of the antitriplet diquark state, which itself has been shown to be equal to that of the $Q \bar{Q}$ singlet state [14], therefore reflecting the properties of the thermal medium rather than that of a particular hadronic system.

Furthermore, we can apply the relations used in [14] to calculate entropy and internal energy from free energies of a $Q Q$ system also for the $Q Q Q$ system. The entropy in the color singlet channel is defined by

$$
S_{Q Q Q}^{1}(P, T)=\frac{\partial F_{Q Q Q}^{1}(P, T)}{\partial T}
$$




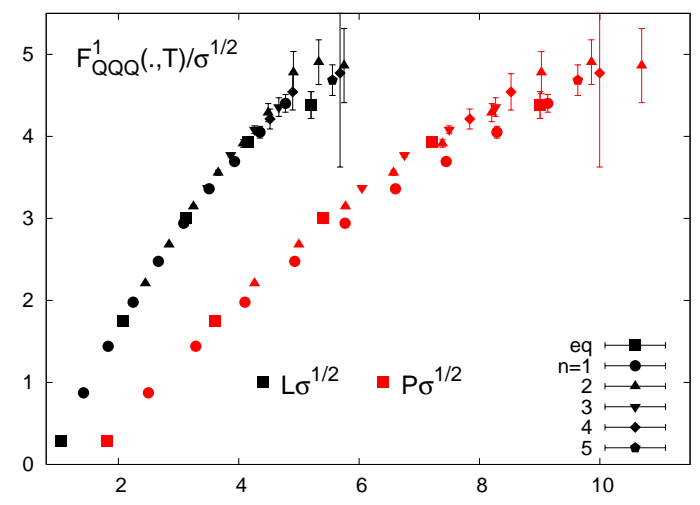

FIG. 9: $F_{Q Q Q}^{1}(., T)$ at $T / T_{c}=0.925$ versus the Y-string length $L$ and the perimeter $P$ for equilateral geometries (eq) and geometries with base length $R_{b} \sqrt{\sigma}=n \sqrt{\sigma} \sqrt{2}$, where $n$ is given in the legend.

and the internal energy in this channel is

$$
U_{Q Q Q}^{1}(P, T)=-T^{2} \frac{\partial F_{Q Q Q}^{1}(P, T) / T}{\partial T}
$$

We then obtain for the entropy

$$
\lim _{P \rightarrow 0} S_{Q Q Q}^{1}(P, T)=0,
$$

i. e. the entropy contribution in the free energy of the $Q Q Q$ singlet color channel vanishes at small distances. Moreover, we find for the internal energy, that

$$
\lim _{P \rightarrow 0} U_{Q Q Q}^{1}(P, T)=\lim _{P \rightarrow 0} V_{Q Q Q}(P),
$$

where $V_{Q Q Q}(P)=\frac{1}{2} \sum_{i<j} V_{Q \bar{Q}}\left(R_{i j}\right)$ and $V_{Q \bar{Q}}(r)$ is the quark-anti-quark potential at $T=0$. This means that the internal energy becomes $T$-independent for small perimeters and thus, together with (30), the singlet free energy itself is $T$-independent for small perimeters, as was already seen in sec. $\mathrm{VA}$. This behavior is already known for $Q \bar{Q}$ singlet free energies and reflects the fact that the baryonic system is less and less effected by the surrounding thermal medium when going to smaller and smaller perimeters.

\section{Free energies below $T_{c}$}

We now examine the $Q Q Q$ free energies below $T_{c}$. We start by looking at the relation between the $Q Q Q$-singlet and the $Q Q$-antitriplet free energies. If $F_{Q Q Q}^{1}$ can be expressed in terms of the sum of $F_{Q Q}^{\overline{3}}\left(R_{i j}, T\right)$ also below $T_{c}$, i. e. if a $\Delta$-ansatz for the flux tube shape together with the same string tension holds, then $F_{Q Q Q}^{1}$ is a function of the perimeter only and $\Delta F(P, T)=$ $F_{Q Q Q}^{1}(P, T)-\sum_{i<j} F_{Q Q}^{\overline{3}}\left(R_{i j}, T\right)$ should be equal to a $T$-dependent constant $k(T)$ for all $P$. In fig. 8 we show

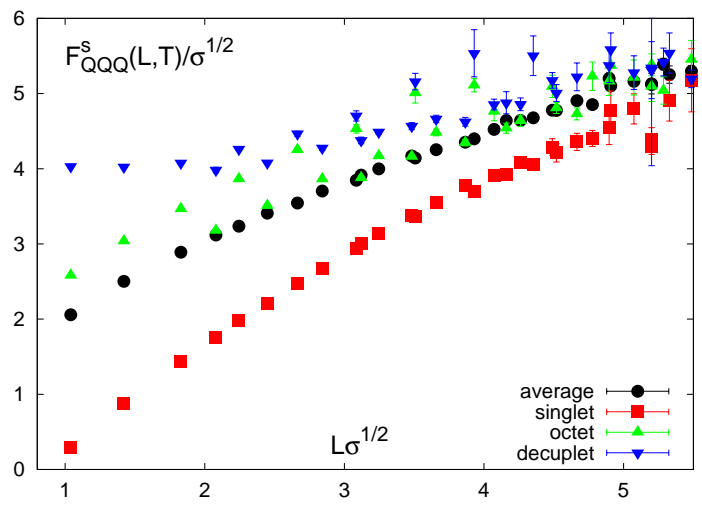

FIG. 10: Free energies of the color channels versus the Ystring length $L$ at $T / T_{c}=0.925$ for all geometries calculated.

$\Delta F(P, T) / T$ for $T<T_{c}$ versus the perimeter, $P$, for different geometries. Here we have set $\Delta F\left(P_{\min }, T\right)=0$, where $P_{\min }$ is the smallest perimeter calculated. We can see clear deviations of $\Delta F(P, T)$ to smaller values, most strongly for the lowest temperatures, deviations from zero becoming smaller with growing temperature. For $T / T_{c}=0.986$ we have $\Delta F(P, T) \approx 0$. Hence, $F_{Q Q Q}^{1}(P, T)$ can not be expressed in terms of the sum of $F_{Q Q}^{3}\left(R_{i j}, T\right)$ except close to the critical temperature. We are left with two possibilities now. First, we could still have a $\Delta$-shaped flux tube, but with a different string tension than that observed for the $Q Q$-antitriplet. Since we see $\Delta F(P, T)<0$, we would expect the string tension to be smaller than in the $Q Q$-antitriplet. In this case the perimeter $P$ would still be the right distance measure for the $Q Q Q$-singlet free energy, i. e. $F_{Q Q Q}^{1}$ should be a smooth function of $P$ for all geometries. Or, secondly, the flux tube is Y-shaped and $L$ is the right distance measure. In this case, $F_{Q Q Q}^{1}$ should be a smooth function of $L$ for all geometries in which a flux tube can form.

To elaborate more on the shape of the flux tube, we take a closer look at the $Q Q Q$-singlet free energy at a particular temperature below $T_{c}$. We analyze the free energy for different geometries as a function of $L$ and $P$. For equilateral triangles a simple geometrical relation exists between the length of a Y-shaped flux tube $L$ and the length of a $\Delta$-shaped flux tube $P$, which is $P=\sqrt{3} L$. Hence for equilateral geometries the $Q Q Q$-singlet free energy is a smooth function in both Ansätze. For more general geometries like the isoscele triangles we calculated, no simple relation between $L$ and $P$ exists. This may help to clarify the situation more directly. Therefore we plot the $Q Q Q$-singlet free energy at $T / T_{c}=0.925$ versus the $\mathrm{Y}$-string length $L$ and the perimeter $P$. This is shown in fig. 9 for equilateral geometries and also for isoscele geometries up to $n=5$ (see sec. IVB). We observe that the $Q Q Q$-singlet free energy is indeed a smooth function of $L$ for all geometries calculated. On the other hand, when the free energy is plotted versus the perimeter $P$ different branches become visible depending on the geometry. 


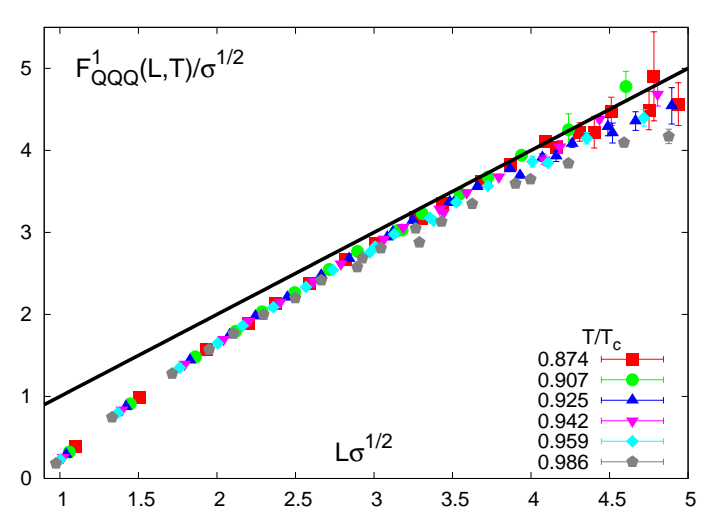

FIG. 11: $F_{Q Q Q}^{1}(L, T)$ below $T_{c}$ versus the Y-string length $L$ for all geometries.

This is most prominent for the $n=1$ triangles. Therefore we find strong hints, that the shape of the flux tube in the $Q Q Q$-singlet system is indeed that of the Y-ansatz and its string length $L$ is the right distance measure for the system.

Having established this, we take a look at the other color channels below $T_{c}$ and analyze whether they display a smooth behavior as function of $L$ as well. In fig. 10 we show the different color channels of the $Q Q Q$-free energy at $T / T_{c}=0.925$ over the $\mathrm{Y}$-string length $L$. We see again that the singlet is the most attractive channel followed by the average free energy, which is also a smooth function of $L$. The octet channel is still attractive, but weaker so than the average free energy. The decuplet free energy is attractive for large $L$ but becomes flat at smaller $L$, possibly hinting at a turnover and at a repulsive behavior at even smaller $L$. Both the decuplet and the octet channel are not smooth functions over either distances $L$ and $P$, but become volatile for $L \sqrt{\sigma} \gtrsim 2$. This suggests that no flux tube forms in these two channels, besides that the octet free energy shows an overall attractive behavior.

Finally, we examine more closely the temperature dependence of $F_{Q Q Q}^{1}$. In fig. 11 we show the renormalised $Q Q Q$-singlet free energy versus $L$ for all temperatures below $T_{c}$. We observe $F_{Q Q Q}^{1}(L, T)$ to nearly coincide at distances $L \sqrt{\sigma} \lesssim 4$ for all temperatures $T / T_{c} \leq 0.959$. We see that at large distances the free energies approach $F(L, T)=\sigma L$ represented by the black line in fig. [1], where $\sigma$ denotes the string tension in a $Q \bar{Q}$-system. The phenomenon of an almost $T$-independent string tension has also been observed in [7], using a fit ansatz in full QCD. As was already seen in fig. 8, at $T / T_{c}=0.986$ we observe deviations to smaller values. This might be due to flux tube broadening setting in near $T_{c}$, leading to an overlapping of the three branches of the Y-shaped flux tube. Filling the space between the three static quarks the separation dependence of the singlet free energy is effectively described by the $\Delta$-Ansatz at these temperatures.

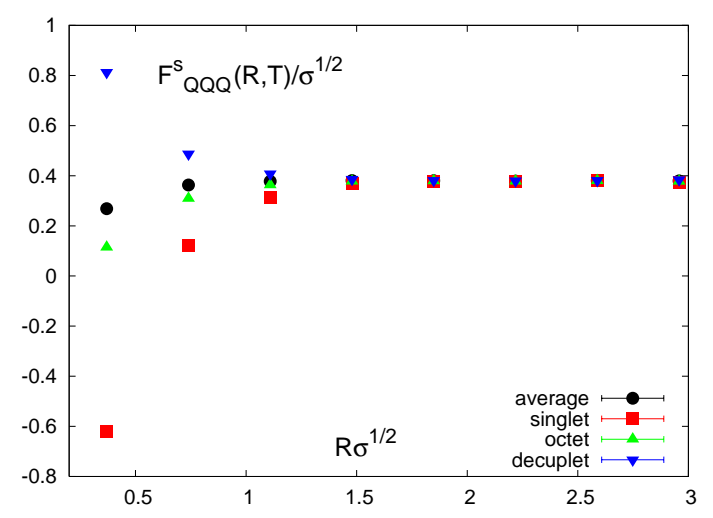

FIG. 12: Free energies in different color channels in 2-flavor QCD from calculations on a $16^{3} \times 4$ lattice at $T / T_{c}=1.99$.

\section{RESULTS IN 2-FLAVOR QCD}

In this section we analyse the $Q Q Q$-free energies obtained on a $16^{3} \times 4$ lattice in 2-flavor QCD with a bare quark mass of $\frac{m}{T}=0.4$. For further details, see sec. [IV] Data for the $Q Q$ free energies is again taken from [14].

\section{A. Color Channels}

In fig. 12 we show the free energies of three quark systems with equilateral geometries in different color channels at $T / T_{c}=1.99$. Like in the pure gauge case, we observe the singlet to be strongly, the octet weaker attractive and the decuplet to be repulsive. For large distances $R$ the free energies in all color channels approach a common value. Again, we obtain similar results for all $T>T_{c}$.

\section{B. Singlet free energy}

We now compare the free energy of the $Q Q Q$-singlet with the free energy of the $Q Q$-antitriplet for 2-flavor QCD. In fig. 13 we show $F_{Q Q Q}^{1}(R, T)$ and $3 F_{Q Q}^{\overline{3}}(R, T)-$ $3 F_{Q}(T)$ versus the edge length $R$ of the equilateral triangles and $Q Q$ distance, respectively. As in pure gauge theory, both curves coincide for all temperatures above $T_{c}$. Thus we find the $Q Q Q$-singlet free energy of equilateral geometries can be described as the sum of three $Q Q$-antitriplet free energies plus self energy terms as well in 2-flavor QCD above $T_{c}$.

Below $T_{c}$ we expect to see string breaking also in the $Q Q Q$-singlet free energy, the breaking mechanism being more involved than in the $Q \bar{Q}$ case [7]. Indeed, except at $T / T_{c}=0.97$, where both quantities agree, we see deviations for the $Q Q Q$-singlet from the simple relation to the $Q Q$ free energies. In fig. 14 we compare $F_{Q Q Q}^{1}(L, T)$ in 2-flavor QCD $\left(T / T_{c}=0.88,0.91\right)$ and pure gauge theory $\left(T / T_{c}=0.874,0.907\right)$ for all geometries calculated. 


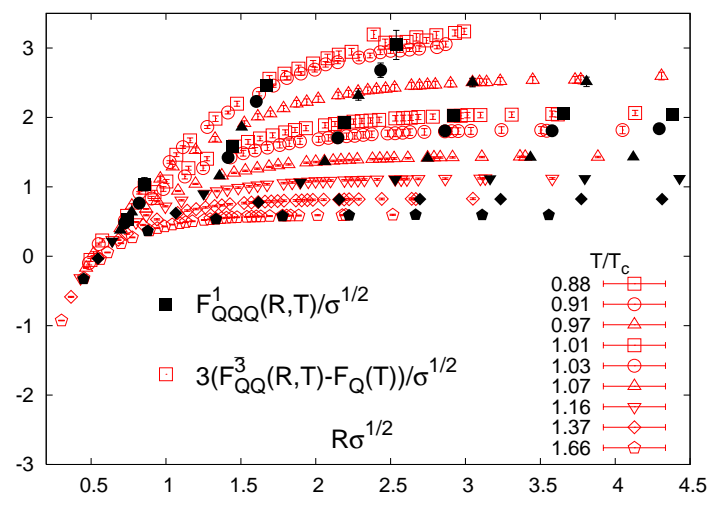

FIG. 13: $F_{Q Q Q}^{1}(R, T)$ and $3\left(F_{Q Q}^{\overline{3}}(R, T)-F_{Q}(T)\right)$ versus $R$, the edge length of the equilateral triangles and $Q Q$-distance, respectively.

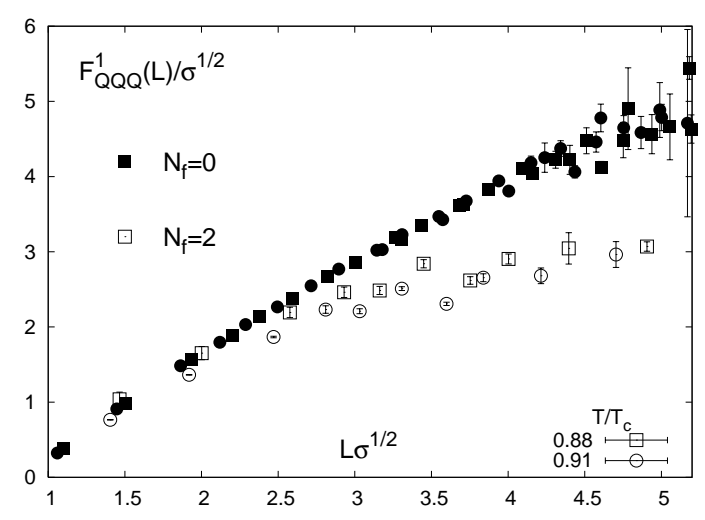

FIG. 14: Comparison of $F_{Q Q Q}^{1}(L, T)$ in 2-flavor QCD (open symbols) and $\mathrm{SU}(3)$ pure gauge theory (filled symbols) for two temperatures below $T_{c}$.

Here the pure gauge data have been obtained from simulations on a $32^{3} \times 4$ lattice. The $N_{f}=2$ free energies start to deviate from the pure gauge result already at short distances to smaller values and eventually become flat. Specifying a definite value for the string breaking distance is quite difficult given the present data. Nevertheless we can give a rough estimate for the distance at which the pure gauge free energies assumes the asymptotic value of the 2 -flavor free energies, which is $L \sqrt{\sigma} \approx 3$. For higher $T$, this distance becomes smaller. The string breaking distance for the $Q \bar{Q}$ singlet free energies has been determined in [24] as the distance where the $T=0$ potential assumes the large distance asymptotic value of the free energy. This lead to values between $2.8 / \sqrt{\sigma}$ for $T / T_{c}=0.874$ and $1.7 / \sqrt{\sigma}$ close to $T_{c}$.

\section{Decuplet free energies}

For the $Q Q Q$-decuplet free energies we show $F_{Q Q Q}^{10}(R, T)$ and $3\left(F_{Q Q}^{6}(R, T)-F_{Q}(T)\right)$ for equilateral

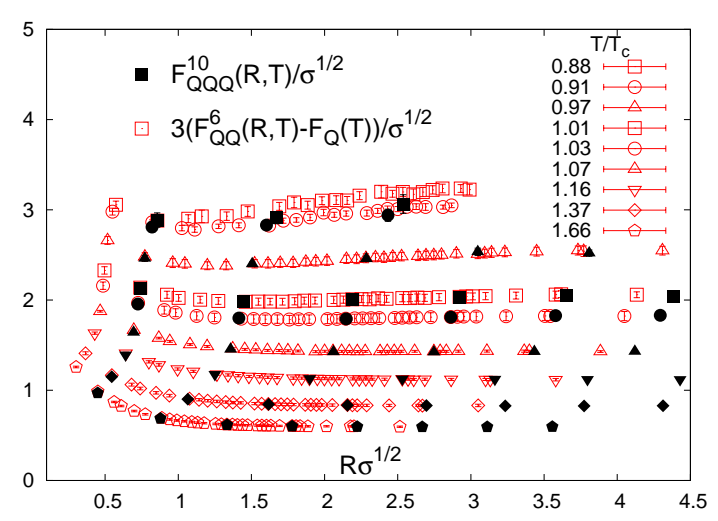

FIG. 15: $\quad F_{Q Q Q}^{10}(R, T)$ and $3\left(F_{Q Q}^{6}(R, T)-F_{Q}(T)\right)$ versus $R$, the edge length of the equilateral triangles and $Q Q$-distance, respectively.

geometries in fig. 15. Like in pure gauge theory, we observe both quantities to coincide for all temperatures above $T_{c}$ and distances $R$. The situation below $T_{c}$ is analogous to the singlet case. At the temperature closest to $T_{c}$ both quantities coincide for all $R$, at smaller $T$ we observe deviations to smaller values for $F_{Q Q Q}^{10}(R, T)$.

Hence we see, that the singlet (decuplet) $Q Q Q$ free energy can be described as a sum of antitriplet (sextet) $Q Q$ free energies and the self energy contributions of the three quarks above $T_{c}$ (Eqs (21) and (24)) also in 2-flavor QCD. Again, we obtain similar plots for $F_{Q Q Q}^{8}(R, T)$ in accordance with (25).

\section{SCREENING OF OCTET AND DECUPLET FREE ENERGIES}

As was shown in 14], the screening of diquark free energies in the anti-triplet and sextet representation at small distances becomes identical to the screening of a single fermion in a color anti-triplet and sextet representation, respectively. This holds for all temperatures in 2-flavor QCD and in the deconfinement phase of SU(3) pure gauge theory.

We will now show, that analogous statements are true in the deconfinement phase for octet free energies of the $Q \bar{Q}$-system and for octet and decuplet free energies of the $Q Q Q$-system, as we have proposed in sec. III.

When combining singlet and octet free energies or singlet and decuplet free energies in proportion to the corresponding Casimir factors (cf. Tab. I) such that the repulsive and attractive Coulombic contributions cancel, we 


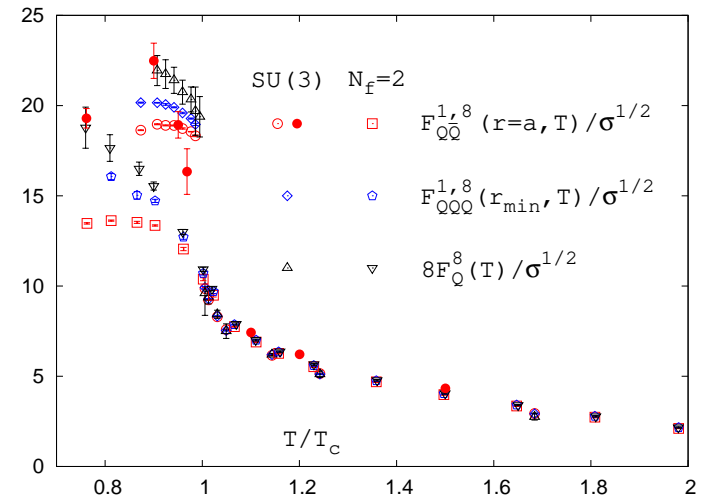

FIG. 16: Comparison of singlet and octet free energies at small distances with the free energy of a static fermion in the adjoint representation. We have used $F_{Q \bar{Q}}^{1,8}(R=a, T)=$ $F_{Q \bar{Q}}^{1}(R=a, T)+8 F_{Q \bar{Q}}^{8}(R=a, T)$ and $F_{Q Q Q}^{1,8}\left(R_{\min }, T\right)=$ $-2 F_{Q Q Q}^{1}\left(R_{\min }, T\right)+8 F_{Q Q Q}^{8}\left(R_{\min }, T\right)$. This particular combinations of $Q \bar{Q}$ - and $Q Q Q$-free energies shown eliminate the short distance Coulomb terms contributing to them according to (34) - (36). Open symbols: $N_{\tau}=4$ data, closed symbols: $N_{\tau}=8$ data. For the $Q Q Q$-free energies we used equilateral geometries with edge length $R_{\min }=\sqrt{2} a$.

expect to find

$$
\begin{aligned}
& \lim _{R \rightarrow 0}\left(F_{Q \bar{Q}}^{(1)}(R, T)+8 F_{Q \bar{Q}}^{(8)}(R, T)\right) \\
= & \lim _{r \rightarrow 0}\left(-2 F_{Q Q Q}^{(1)}(R, T)+8 F_{Q Q Q}^{(8)}(R, T)\right) \\
= & 8 F_{Q}^{(8)}(T)
\end{aligned}
$$

and

$$
\lim _{R \rightarrow 0}\left(F_{Q Q Q}^{(1)}(R, T)+2 F_{Q Q Q}^{(10)}(R, T)\right)=2 F_{Q}^{(10)}(T)
$$

where $R$ stands for the edge length of the equilateral geometries in the case of $Q Q Q$ free energies; $F_{Q}^{(8)}(T)$ and $F_{Q}^{(10)}(T)$ are the free energies of a static quark in a color octet and decuplet representation, respectively. The latter are given by the corresponding Polyakov loop expectation values,

$$
\mathrm{e}^{-F_{Q}^{(D)} / T}=\left\langle L_{D}(\mathbf{x})\right\rangle
$$

where $D=8,10$ and

$$
\begin{aligned}
L_{8}(\mathbf{x}) & =\frac{1}{8}\left(9|L(\mathbf{x})|^{2}-1\right) \\
L_{10}(\mathbf{x}) & =\frac{1}{10}\left(27 L(\mathbf{x})^{3}-18|L(\mathbf{x})|^{2}+1\right)
\end{aligned}
$$

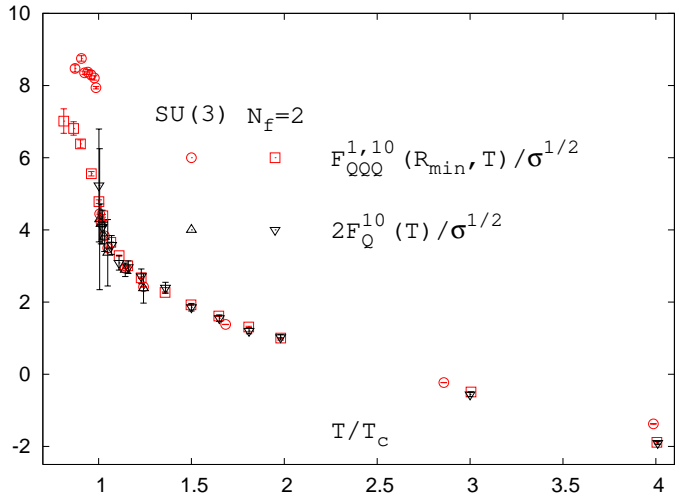

FIG. 17: Comparison of singlet and decuplet free energies at small distances with the free energy of a static fermion in the decuplet representation. We have used $F_{Q Q Q}^{1,10}\left(R_{\min }, T\right)=$ $F_{Q Q Q}^{1}\left(R_{\min }, T\right)+2 F_{Q Q Q}^{10}\left(R_{\min }, T\right)$. This particular combination of $Q Q Q$-free energies shown eliminates the short distance Coulomb terms contributing to them accroding to (37). For the $Q Q Q$-free energies we used $N_{\tau}=4$ data and again equilateral geometries with edge length $R_{\min }=\sqrt{2} a$.

are obtained through group theoretical relations. The Polyakov loops $\left\langle L_{8}\right\rangle$ and $\left\langle L_{10}\right\rangle$ have been computed in [27]. We note here, that these quantities - unlike the Polyakov loop in the fundamental representation - do not have to vanish in the confinement phase of $\mathrm{SU}(3)$ pure gauge theory due to their vanishing triality.

(34) - (37) are indeed fulfilled for all temperatures above $T_{c}$ as can be seen from Figs. 16] and 17. In particular, the combinations of singlet and octet free energies of the $Q \bar{Q}$ - and the $Q Q Q$-system coincide in the deconfinement phase (35), thus showing the screening at small distances to depend only on the color representation and not on the particular static quark system under consideration. Below $T_{c}$ deviations become apparent in Fig. 16. The reason probably is, that the minimal distances used in the present analysis $(R=a$ for the $Q \bar{Q}$-system and $R_{\text {min }}=\sqrt{2} a$ for the $Q Q Q$-system respectively) are still too large at small temperatures to be a good approximation for the small distance limit in (34) - (37). In fact, the $N_{\tau}=8$ data, where the lattice spacing $a$ is only half as large at the same value of the temperature, agree much better at least close to $T_{c}$.

\section{CONCLUSIONS}

We have calculated the free energy of static three quark systems in different color channels in the quenched approximation and in 2-flavor QCD at finite temperature. We have shown that above the critical temperature the singlet and decuplet free energies of the three quark system can be described by the sum of the free energies of the corresponding diquark system plus self energy contributions. Therefore the screening of the singlet $Q Q Q$ free energies is comparable to that of the $Q Q$ free en- 
ergies reflecting the screening properties of the thermal medium. Below $T_{c}$ we found evidence for a $\mathrm{Y}$-shaped flux tube in the quenched approximation for the singlet and color average channel. The string tension agrees with that deduced from $Q \bar{Q}$-systems at $T=0$ and has been found to be independent of temperature for $T / T_{c} \lesssim 0.959$ in the singlet color channel. In 2-flavor QCD we observed string breaking in the $Q Q Q$ singlet free energies at distances around $3 / \sqrt{\sigma}$. Moreover, we were able to show that at short distances the screening properties of octet free energies of the $Q \bar{Q}$ - and the $Q Q Q$-system and the decuplet free energies of the $Q Q Q$-system coincide with those of a single static quark in the corresponding color state above $T_{c}$.

\section{Acknowledgment}

This manuscript has been authored under Contract No. DE-AC02-98CH10886 with the U. S. Department of Energy. O.V. and at an early stage K.H. have been supported by the Deutsche Forschungsgemeinschaft (DFG) under grant GRK 881.
[1] R. Sommer and J. Wosiek, Nucl. Phys. B 267, 531 (1986)

[2] G. S. Bali, Phys. Rept. 343, 1 (2001)

[3] C. Alexandrou, P. De Forcrand and A. Tsapalis, Phys. Rev. D 65, 054503 (2002)

[4] T. T. Takahashi, H. Suganuma, Y. Nemoto and H. Matsufuru, Phys. Rev. D 65, 114509 (2002)

[5] T. T. Takahashi and H. Suganuma, Phys. Rev. D 70, 074506 (2004)

[6] V. G. Bornyakov et al. [DIK Collaboration], Phys. Rev. D 70 (2004) 054506

[7] V. G. Bornyakov et al., Prog. Theor. Phys. 112, 307 (2004)

[8] Ph. de Forcrand and O. Jahn, Nucl. Phys. A 755, 475 (2005)

[9] D. S. Kuzmenko and Y. A. Simonov, Phys. Lett. B 494, $81(2000)$

[10] J. Liao and E. V. Shuryak, Nucl. Phys. A 775, 224 (2006)

[11] S. Nadkarni, Phys. Rev. D 34, 3904 (1986)

[12] S. Nadkarni, Phys. Rev. D 33, 3738 (1986)

[13] J. M. Cornwall, Phys. Rev. D 54, 6527 (1996)

[14] M. Döring, K. Hübner, O. Kaczmarek and F. Karsch,
Phys. Rev. D 75, 054504 (2007)

[15] O. Kaczmarek, F. Karsch, P. Petreczky and F. Zantow, Phys. Lett. B 543, 41 (2002)

[16] P. Weisz, Nucl. Phys. B 212, 1 (1983)

[17] P. Weisz and R. Wohlert, Nucl. Phys. B 236, 397 (1984)

[18] F. Karsch, E. Laermann and A. Peikert, Nucl. Phys. B 605, 579 (2001)

[19] C. R. Allton et al., Phys. Rev. D 66, 074507 (2002)

[20] C. R. Allton et al., Phys. Rev. D 68, 014507 (2003)

[21] B. Beinlich, F. Karsch and A. Peikert, Phys. Lett. B 390, 41 (1997)

[22] O. Philipsen, Phys. Lett. B 535, 138 (2002)

[23] O. Jahn and O. Philipsen, Phys. Rev. D 70, 074504 (2004)

[24] O. Kaczmarek and F. Zantow, Eur. Phys. J. C 43, 63 (2005)

[25] O. Kaczmarek, S. Gupta and K. Hübner, PoS(LATTICE 2007)195 arXiv:0710.2277 [hep-lat].

[26] O. Kaczmarek, PoS C POD07, 043 (2007)

[27] S. Gupta, K. Hübner and O. Kaczmarek, in preparation 